\title{
Predicting the Actual Location of Faults in Underground Optical Networks using Linear Regression
}

\author{
Adebayo Adekoya ${ }^{1}$, Owusu Nyarko-Boateng ${ }^{2}$, and Benjamin Weyori ${ }^{1}$ \\ ${ }^{1}$ University of Energy and Natural Resources \\ ${ }^{2} \mathrm{UENR}$
}

July 20, 2020

\begin{abstract}
Optical cables are enormous transmission media which carries high-speed data across transatlantic, intercontinental, international boundaries and cities. The optical cable is essential in data communication. The cable has become an indispensable component in optical communications infrastructure; hence, conscious efforts are always adopted to prevent or minimize faults in the optical network infrastructure. Typically, tracing fault in the underground optical network has been difficult even though optical time-domain reflectometer (OTDR) has been used to measure the distance of faults in the underground fiber cable. The methodologies deployed in the reviewed literature indicate a vast gap between the fault distance measured by the OTDR and the actual distance of fault. This paper observed the difficulties involved in tracing the actual spot of fault in the underground optical networks. The difficulty of tracing these underground faults mostly result in an undue delay and loss of revenue. This research presents a machine learning (ML) approach to predict the actual location of a fiber cable fault in an underground optical transmission link. Linear regression in the python sci-kit learn library was used to predict the actual location of a fault in an underground optical network. The MSE and MAE evaluation matrix used provided good accuracy results of 0.061291 and 0.080143 , respectively. The result obtained in this paper indicates that faults in underground optical networks can be found quickly to avoid the delays in the fault tracing process, which leads to an excessive revenue loss.
\end{abstract}

\section{Hosted file}

Manuscript_main.docx available at https://authorea.com/users/319288/articles/471023predicting-the-actual-location-of-faults-in-underground-optical-networks-using-linearregression 